\title{
Polarimetry of Exoplanetary System CoRoT-2
}

\author{
N. M. Kostogryz, T. M. Yakobchuk, and A. P. Vidmachenko \\ Main Astronomical Observatory of NAS of Ukraine, \\ 27, Zabolotnoho str., Kyiv 03680, Ukraine \\ email: kosn@mao.kiev.ua yakobchuk@mao.kiev.ua
}

\begin{abstract}
We present the results of modelling the polarization resulting from the planetary transits and stellar spots in the system Corot-2 using the Monte Carlo method. The planetary transit was estimated to produce a polarization maximum at the limb of $\sim 5 \times 10^{-6}$, adopting solar center-to-limb polarization. Assuming different parameters of the spots, we evaluated the flux and polarization changes due to the stellar activity.
\end{abstract}

Keywords. methods: numerical, techniques: polarimetric, planetary systems, stars: spots

\section{Introduction}

It is known that radiation from an unresolved, centro-symmetric star is normally unpolarized as the average of the polarization directions of all photons from the star detected by the observer results in zero polarization. However, an intrinsic polarization can occur if the star is not centro-symmetric, e.g. during planetary transit. This effect is also known as the Chandrasekhar effect (Chandrasekhar 1950). In order to model it we used the Monte Carlo method as proposed by Carciofi \& Magalhães (2005). For a known set of parameters that describe the configuration of the star-planet system, we adopted a photon-by-photon procedure with weights according to the chosen point on the stellar surface. If the photon packet was emitted in the opposite hemisphere with respect to the observer or its trajectory crossed the planet, the weight was set to zero. Otherwise, the weight was chosen according to the limb-darkening law (see also Kostogryz et al. 2011). The spot weights were calculated differently, accounting for their lower temperature.

\section{Results and discussions}

We considered a young and active star Corot-2, the second planet-hosting star discovered by the CoRoT satellite. It has a highly active and intrinsically variable host star. Its rotation period is $P_{\text {star }}=4.52 d$ (Lanza et al. 2009), while the orbital planetary period is only three time less $\left(P_{p l}=1.74 d\right)$. Being interested in the occultation effects, we particularly chose the system for the high ratio of planetary to star radii $R_{\text {planet }} / R_{\text {star }}$ $=0.167$ (Schneider 2011).

The upper panels in Figure 1 illustrate the size of the planet compared to the star and the path of the transit across the stellar disk. For cases b) and c), positions and radii of the spots are shown. The lower panels present the time dependence of the stellar flux, Stokes parameters and polarization degree. The maximum polarization observed on the stellar limb is estimated at $\sim 5 \times 10^{-6}$ (Fig. 1a). While the shapes of the curves for polarization parameters remain essentially the same, the average values grow considerably when spots are added (Fig. 1b). The planet's contribution to the polarization also dominates in one stellar rotation period, as is seen from Fig. 1c. 

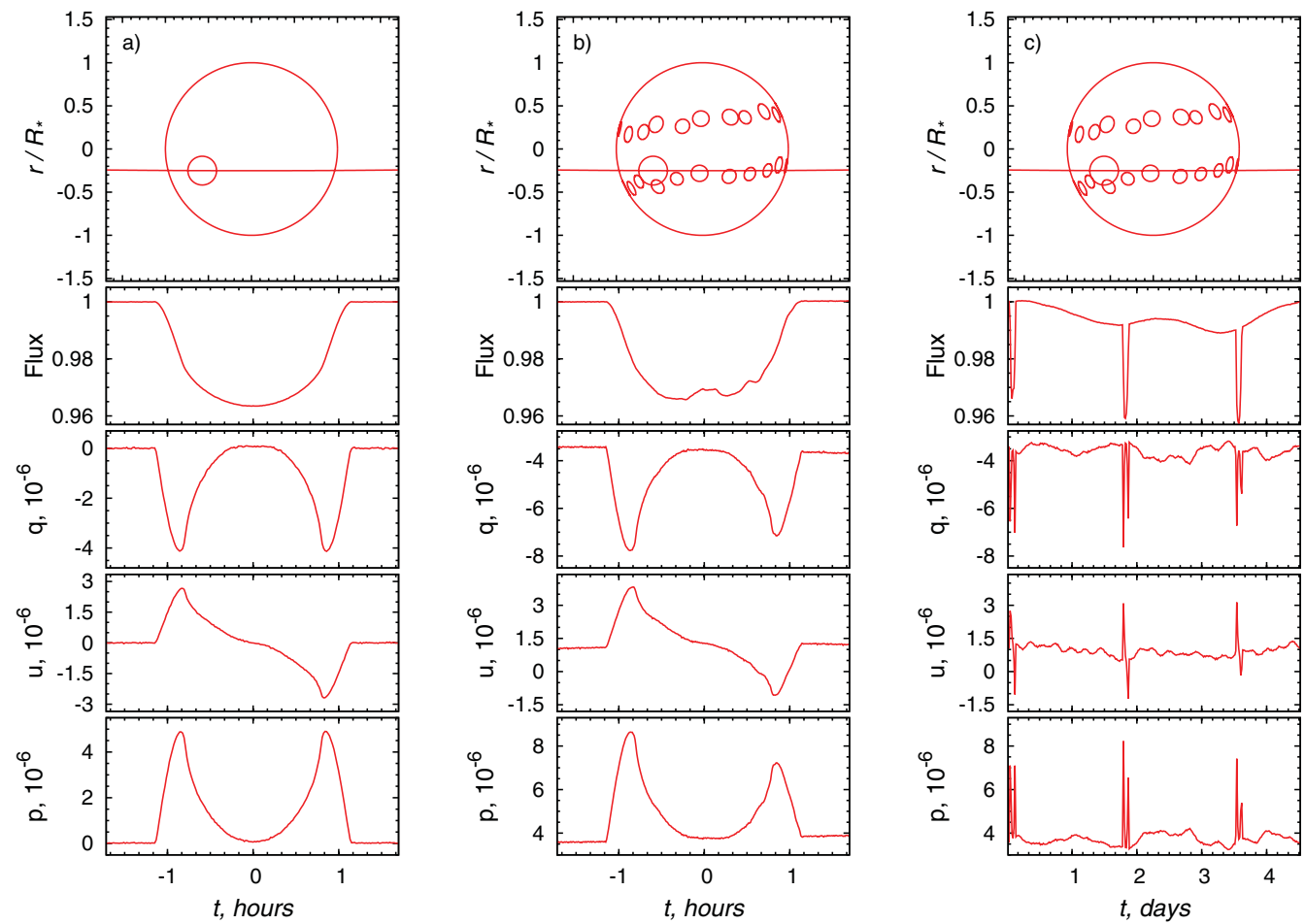

Figure 1. Modelled curves for the fluxes and polarization parameters for CoRoT-2: a) one planet transit; b) one planet transit with stellar spots; c) planet transits and spots for one stellar rotation period.

\section{Conclusions}

In this study the two possible cases of symmetry breaking of the host star Corot-2 were investigated. The first one appears as a result of an occultation of the star by a transiting planet; the second one is the total effect of the planetary transit and spots on the stellar surface. The planetary transit was found to produce a polarization maximum at the limb of $\sim 5 \times 10^{-6}$, adopting the solar center-limb polarization. At the same time, it was shown that the polarization value can vary substantially depending on the positions and sizes of the stellar spots.

\section{Acknowledgement}

The authors gratefully acknowledge Dr. Sci. Nataliya Shchukina for providing the data on solar polarization. This work has been partially funded by National Academy of Sciences of Ukraine through project 1.4.6/5-261B.

\section{References}

Chandrasekhar, S. 1950, Radiative Transfer. Dover Press, New York

Carciofi, A. C. \& Magalhães, A. M. 2005, ApJ, 635, 570

Kostogryz N. M., Yakobchuk T. M., Vid'machenko A. P. \& Morozhenko, O. V. 2011, MNRAS, 415,695

Lanza, A. F., Pagano, I., Leto, G., Messina, S., et al., 2005, A\& A, 493, 193

Schneider, J. 2011, on-line http://exoplanet.eu 